\title{
Towards image denoising in the latent space of learning-based compression
}

\author{
Michela Testolina, Evgeniy Upenik, and Touradj Ebrahimi \\ Multimedia Signal Processing Group (MMSPG) \\ Ecole Polytechnique Fédérale de Lausanne (EPFL) \\ CH-1015 Lausanne, Switzerland \\ Email: firstname.lastname@epfl.ch
}

\begin{abstract}
In recent years, learning-based image compression has demonstrated similar or superior performance when compared to conventional approaches in terms of compression efficiency and visual quality. Typically, learning-based image compression takes advantage of autoencoders, which are architectures consisting of two main parts: a multi-layer neural network encoder and its dual decoder. The encoder maps the input image represented in the pixel domain to a compact representation, also known as latent space. Consequently, the decoder reconstructs the original image in the pixel domain from its latent representation, as accurately as possible. Traditionally, image processing algorithms, and in particular image denoising, are applied to images in the pixel domain before compression, and eventually in some cases as a post-processing stage after decompression. In this context, the combination of denoising operations with the autoencoder might reduce the computational cost while achieving similar performance in accuracy. In this paper, the idea of combining the image denoising task with compression is examined. In particular, the integration of denoising convolutional layers in the decoder of a learning-based compression network is investigated. Results show that, while the rate-distortion performance of the method is slightly reduced, a gain in the computational complexity can be achieved.
\end{abstract}

Keywords: Image denoising, learning-based compression, compressed domain denoising, latent space, image processing

\section{INTRODUCTION}

Following the constant growth in the total number of images captured and stored on digital devices every year, new image compression solutions are continuously investigated. Recently, learning-based image compression solutions have demonstrated competitive or even superior performance when compared to conventional methods. ${ }^{1-5}$ Learning-based methods are typically based on autoencoders, i.e. neural networks composed of two main components: an encoder that maps the input image into a compressed or latent representation, and a decoder that reconstructs the original image from its latent representation, as accurately as possible.

In a typical camera pipeline, the compression method is generally anticipated and followed by two image processing stages, including a number of the operations that vary according to the model and brand of the camera. In general, the pre-processing includes operations such as demosaicing, white balance adjustment, gamma correction and denoising. The post-processing, on the other hand, usually aims at mitigating the distortions introduced by the compression, including color corrections, sharpening and denoising. The typical camera pipeline is visually presented in Figure 1.

In this context, combining the denoising operations performed before and after the compression in the coding workflow might decrease the computational complexity, and, if optimized for a specific task, ideally even improve the performance. Let us assume the case where the operations are applied on the decoder side to the compressed (or latent) representation of the images. One may train the pipeline with an appropriate strategy, that allows the encoder to learn a compressed representation suitable for the two tasks of decompression and denoising of the latent space. Such types of approaches, in fact, are gaining interest in the standardization communities, for example in the Joint Photographic Experts Group (JPEG) committee. To be precise, in the framework of the JPEG AI project, a requirement has been included for a future learning-based image coding standard to have the 


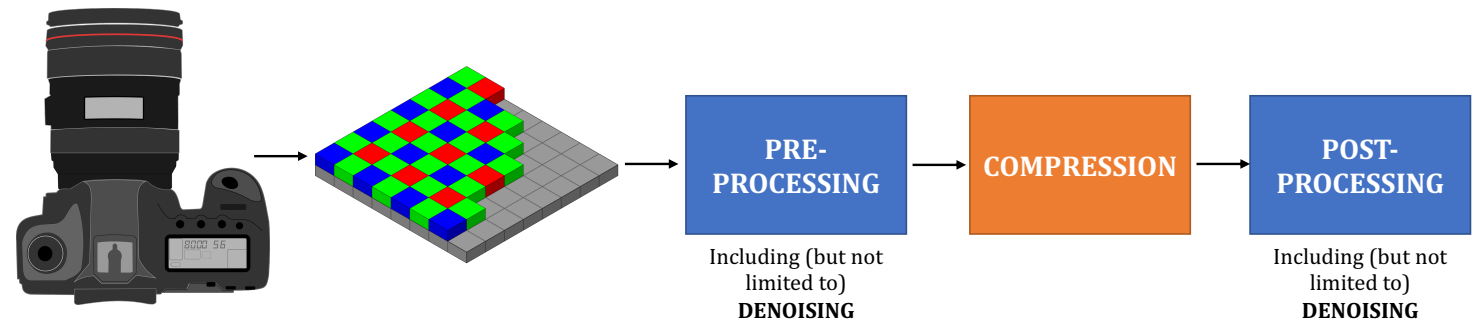

Figure 1: Typical camera pipeline. The light is captured through the lenses and is collected on the image sensor. Then the image is pre-processed with multiple operations (the procedure may differ from camera to camera), where denoising is generally always included. The image is then compressed, and again post-processed with different operations, including denoising.

adaptability of the network to perform image processing and computer vision tasks in the compressed domain, and therefore without the need of a decoder*.

Recently, a number of research works have demonstrated the feasibility of applying computer vision and image processing tasks directly to the compressed or latent representation of images, showing improvements in computational complexity while obtaining similar performance. As an example, Torfason et al. ${ }^{6}$ analyzed the task of image classification and semantic segmentation applied directly in the compressed domain, and demonstrated that it is possible to improve the run-time, memory usage, robustness and synergy, compromising only the performance at the lowest bitrate. In a similar way, Zhang et al. ${ }^{7}$ proposed a content-based image retrieval pipeline, where the compressed bitstreams are used as an input to a learning-based retrieval network. The authors also proposed to jointly train the compression and retrieval pipelines, to achieve the best performance. To the best of our knowledge, the problem of image denoising integrated into the encoder and decoder of learningbased image compression algorithms, and therefore applied to the latent representations, has not been addressed yet.

In this paper, we propose a combined learning-based compression and denoising architecture, which is able to execute both image processing tasks simultaneously. For this purpose, a number of denoising layers have been combined with the decoder, and a novel learning strategy has been adopted. The performance of the proposed architecture has been compared to the pipeline including compression and denoising in cascade, and with the performance of the denoising network without any compression.

The remainder of this paper is organized as follows: Section 2 describes the adopted learning-based compression and denoising architecture; Section 3 presents the proposed combined compression and denoising model, along with the adopted training procedure; in Section 4 the results are presented; finally conclusions are drown in Section 5.

\section{RELATED WORK}

As introduced above, the state of the art of image processing and computer vision methods applied to the compressed or latent representation of images is not yet in a mature form. ${ }^{6,7}$ Instead, multiple methods have been proposed for the tasks of learning-based compression and denoising separately.

Learning-based image compression methods have recently emerged as powerful tools in the context of image compression, capable, in some cases, of outperforming the conventional methods. Among the first works, Toderici et al. ${ }^{8,9}$ proposed a compression framework for thumbnails and image compression, based on Recurrent Neural Networks (RNN). A different approach adopting autoencoder architectures employing Convolutional Neural Networks (CNNs) has been explored in many recent works. ${ }^{2,3,10,11}$ Other more recent methods take advantage of Generative Adversarial Networks (GANs) ${ }^{4,5}$ to create images with a higher level of details. Among the methods presented in this section, the architecture proposed by Ballè et al. ${ }^{2}$ lately became well-known in the learning-based image compression research community, and is even considered a groundbreaking work for its

\footnotetext{
*http://ds.jpeg.org/documents/jpegai/wg1n90021-REQ-JPEG_AI_Use_Cases_and_Requirements.pdf
} 
remarkable performance in terms of visual quality of the decompressed images. For this reason, this architecture has been used as a starting point for our experiments.

Multiple learning-based methods have also been proposed that are targeting the image denoising task. Historically, the first work was published by Jain et al. in $2008,{ }^{12}$ who advocated an architecture based on a five-layer convolutional neural network. Subsequently, other novel and more powerful methods have been proposed, showing increased performance for such types of methods. As an example, Zhang et al. presented a feed-forward convolutional architecture, known as DnCNN, ${ }^{13}$ and successively a similar architecture able to denoise images with a wider range of noise distributions and intensities, known as FFDNet or FDnCNN. ${ }^{14}$ A different approach was investigated by Tai et al., ${ }^{15}$ who proposed a very deep persistent memory network, also known as MemNet, for multiple tasks, among which denoising. This network achieves better performance than the DnCNN, but it is more expensive in terms of computational complexity. A faster solution was proposed by Gu et al., ${ }^{16}$ attaining a good trade-off between computational complexity and visual results. In this paper, the DnCNN and FDnCNN ${ }^{13,14}$ have been employed as guidelines for the proposed combined compression and denoising network, and will be analyzed in more details in the next subsections.

\subsection{Learning-based image coding solution}

As it was already underlined, the proposed combined architecture is based on an existing learning-based solution, and the variational hyperprior model proposed by Ballé et al. ${ }^{2}$ has been selected. This autoencoder solution is an end-to-end convolutional neural network model based on a variational autoencoder that incorporates side information in the form of a hyperprior, able to capture the spatial dependencies in the compressed domain. The loss function handles the trade-off between the bitrate and the distortions on the images, and can be optimized for different objective metrics, i.e. Mean Squared Error (MSE) or Multi-scale Structural Similarity Index (MS-SSIM). In our work, the model optimizing for MSE was used as a reference.

\subsection{Learning-based image denoising architecture}

The DnCNN and FDnCNN ${ }^{13,14}$ were selected as a baseline in our work, due to the fact that they guarantee a suitable balance between performance and computational complexity. The selected models are based on a convolutional neural network architecture composed of a series of convolutional layers in a cascade with ReLU activation and batch normalization. Two different implementations have been used: for the DnCNN, the original code presented by the authors ${ }^{\dagger}$, implemented in Matlab, has been used; for the FDnCNN, the Python implementation is used ${ }^{\ddagger}$.

\subsection{Artificial noisy dataset}

A large-scale dataset containing both noisy images and their corresponding original uncompressed and noisefree version is required for the training of the proposed method. The state of the art lacks a dataset with the above mentioned characteristics. Therefore a synthetic, but realistic noise was applied to a dataset of noisefree uncompressed images. In order to simulate the noise, the method proposed by Foi et al. ${ }^{17}$ was adopted. Specifically, an additive noise with Gaussian-Poissonian distribution was simulated in the images as:

$$
z(x)=y(x)+\sigma(y(x)) \xi(x)
$$

where $z(x)$ represents the noisy image, $y(x)$ the original noise-free image and $\sigma(y(x)) \xi(x)$ the noise component, and:

$$
\sigma(y(x)) \xi(x)=\eta_{p}(y(x))+\eta_{g}(x)
$$

Therefore, the additive noise is composed of two main components:

- a Poissonian component $\eta_{p}(y(x))$, signal-dependent;

\footnotetext{
${ }^{\dagger}$ https://github.com/cszn/DnCNN

${ }^{\ddagger}$ https://github.com/cszn/KAIR
} 

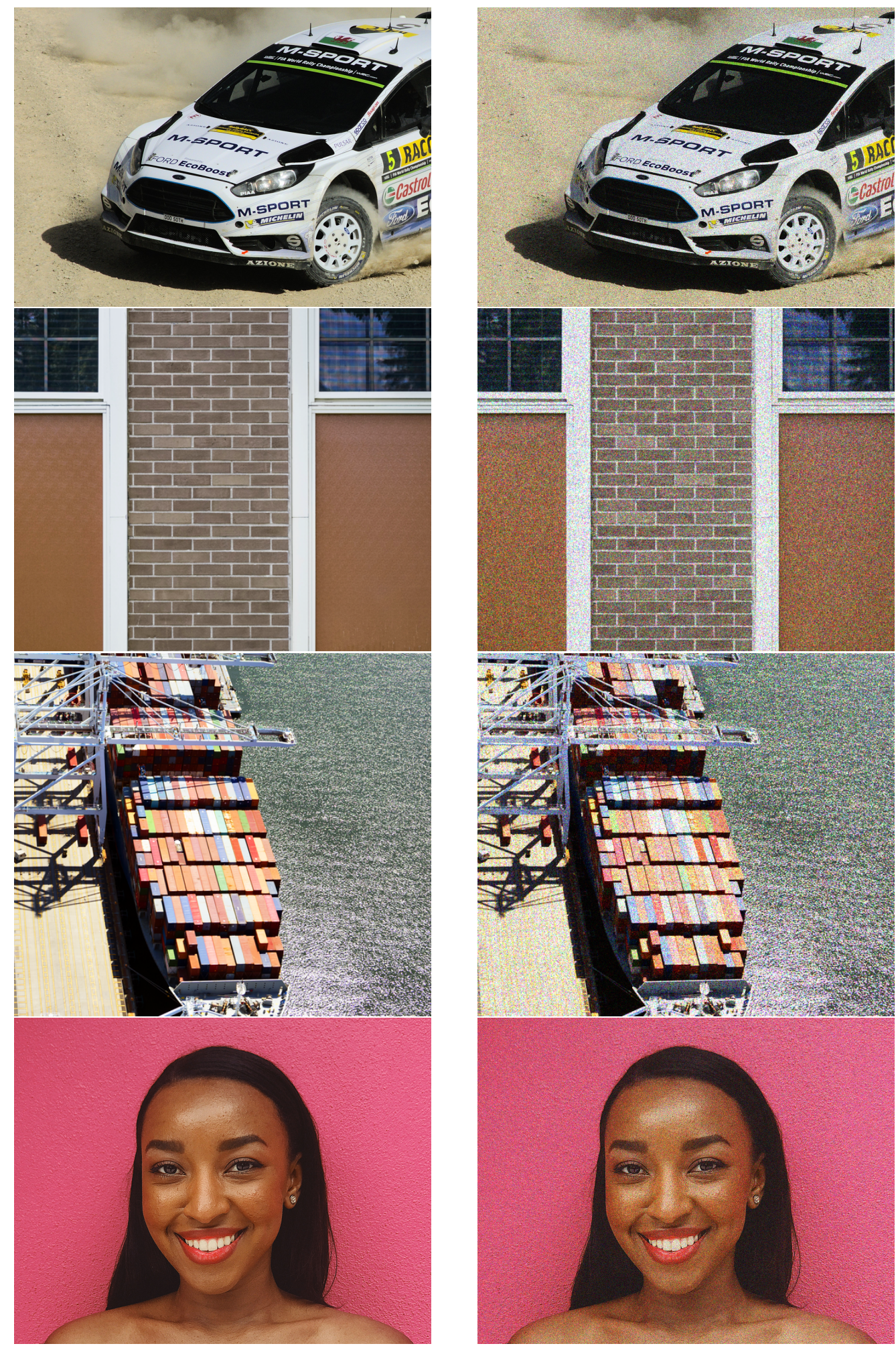

Figure 2: Examples of original uncompressed noise-free images (left column) and the same images in which the artificial noise was simulated, using the approach proposed by Foi et al. ${ }^{17}$ (right column). 


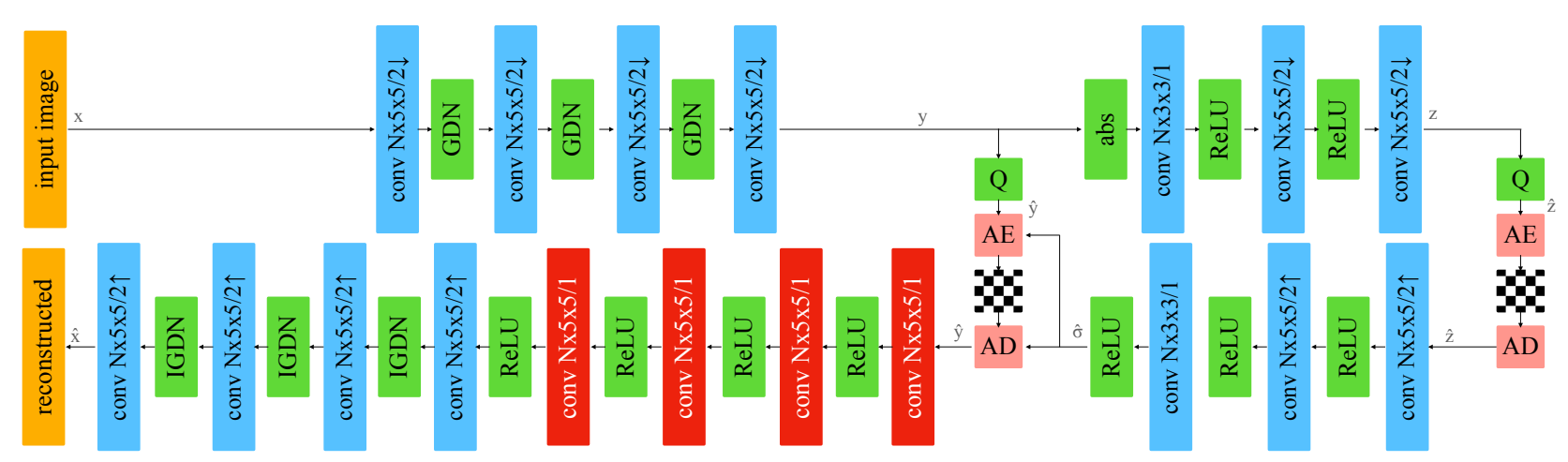

Figure 3: Proposed architecture for combined denoising and compression task. The autoencoder solution proposed by Ballé et al. ${ }^{2}$ was extended with several convolution layers inspired by the DnCNN architecture proposed by Zhang et al. ${ }^{13}$

- a Gaussian component $\eta_{g}(x)$, signal-independent.

The training set was created by applying the described noise to the CLIC training set ${ }^{\S}$, which includes 1,633 training images, both in RGB and grayscale formats, and 102 images as a validation subset. Moreover, the JPEG AI test set $\mathbb{I}$ has been selected for the experiments, and the results on 10 uncompressed RGB 8-bit images with sizes varying from $1336 \times 872$ to $3680 \times 2456$ were analyzed.

Some visual examples of original uncompressed noise-free and noisy images, which are a part of the selected test set, are shown in Figure 2.

\section{COMBINED COMPRESSION AND DENOISING}

Taking inspiration from the learning-based compression and denoising methods that have been presented in Sections 2.1 and 2.2, a novel architecture has been designed by integrating both the compression and denoising operations in the same network. In this section, the proposed combined compression and denoising solution is presented.

\subsection{Combined architecture}

The proposed combined denoising and compression method was designed on the basis of the autoencoder solution by Ballé et al. ${ }^{2}$ The encoder adopted in the proposed architecture is identical to that proposed by Ballé et al., consisting of a number of convolutional layers with GDN activation. On top of this, four additional convolutional layers were combined with the decoder, with ReLU activation function, which should compensate the added complexity introduced by the denoising task, similar to how it was implemented in the DnCNN denoising solution. ${ }^{13}$ The architecture also includes a hyperprior to capture the spatial dependencies in the compressed domain, employed by an arithmetic encoder.

The choice of introducing the denoising layers on the decoder side originates from the idea that image processing operations might be applied to the latent representation of images. If properly learned, they may even benefit from such approach. The encoder, in fact, may specialize in a representation beneficial for a specific task, and the performance of the pipeline may be improved. Based on this observation, the Joint Photographic Experts Group (JPEG) has organized several exploration experiments to assess the validity of this hypothesis. This work can be intended as a first step towards such a type of approaches for the specific task of image denoising.

Figure 3 shows the presented architecture.

\footnotetext{
${ }^{\S}$ https://www.tensorflow.org/datasets/catalog/clic

$\mathbb{I}_{\text {https: //jpegai.github.io/3-datasets/ }}$
} 


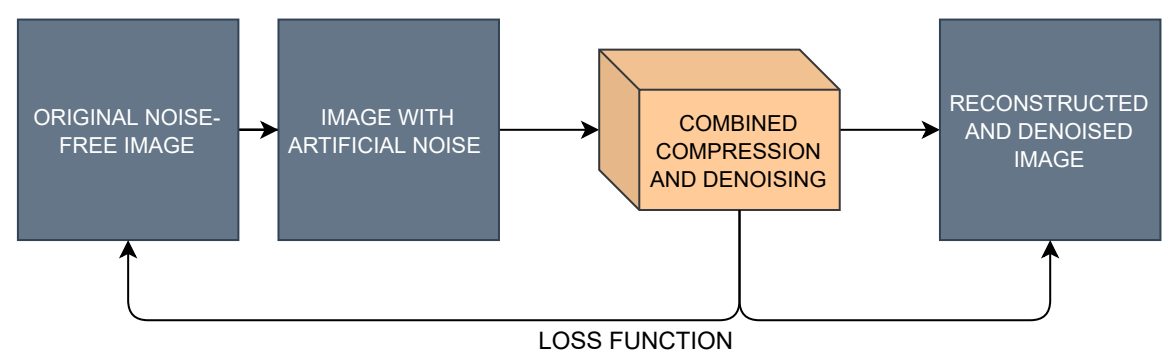

Figure 4: Graphic representation of the proposed training procedure. An artificial but realistic noise is applied to the dataset of uncompressed noise-free images, and the noisy images are used as input to the network. A fullreference objective quality metric is computed between the reconstructed image and the original uncompressed image and optimized as part of the loss function. The selected full-reference objective quality metric in the paper is the MSE.

\subsection{Training procedure}

In order to train the combined denoising and compression architecture, a novel training strategy has been adopted. Since both the original noise-free images and the corresponding noisy images are available, they were both used in the training procedure. Therefore, a full-reference objective quality metric is optimized as part of the loss function.

Specifically, the noise has been applied to the training dataset as presented in Section 2.3, and the obtained noisy images have been fed as an input to the autoencoder. The loss function has therefore been computed between the reconstructed image and the original noise-free image. The model has been optimized for the MSE, and therefore the pixel by pixel difference between the reconstructed and the original noise-free images is optimized. The training procedure is schematically represented in Figure 4.

Four different models have been trained, targeting different bitrates, specifically between 0.2 and 1.5 bits per pixel (bpp), achieved by selecting lambda values of $0.1,0.25,0.4$ and 0.55 . A random patch size of 256 was used for the training and validation set. Moreover, the Adam optimization was used, with a learning rate of 0.0001.

\section{EXPERIMENTAL RESULTS AND DISCUSSION}

To assess the results, the rate-distortion performance of the proposed method has been evaluated. The ratedistortion plots for the PSNR and the MS-SSIM objective quality metrics for four of the test images are shown in Figure 5 and 6.

The results of the proposed method have been compared with the performance of two other pipelines:

- To simulate the ideal condition, the DnCNN denoising architecture was applied to the noisy images, without applying any compression.

- The images obtained by compressing and successively denoising, in separate stages, were also compared to the proposed combined method. The compression was performed using the pre-trained model for the variational autoencoder, ${ }^{2}$ optimized for the MSE, and the denoising using the DnCNN and FDnCNN denoiser. In particular, for the DnCNN the original implementation proposed by the authors, and available in Matlab, was used, and for the FDnCNN a different implementation, available in Python and taking advantage of the graphics processing unit (GPU), has been used. The plots present the results for both implementations. 

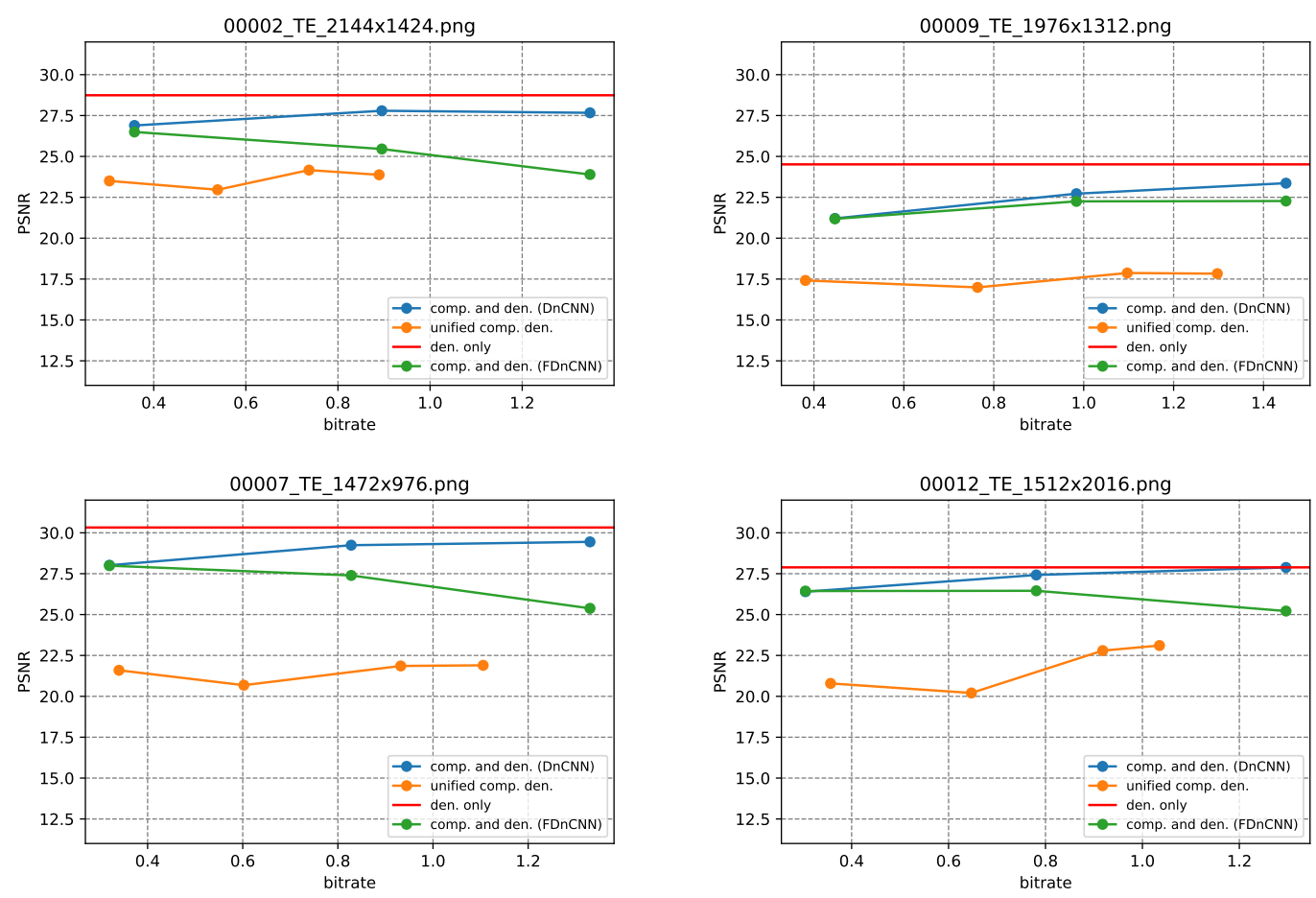

Figure 5: Rate-distortion results for the PSNR objective quality metric on the different compression and denoising pipelines.
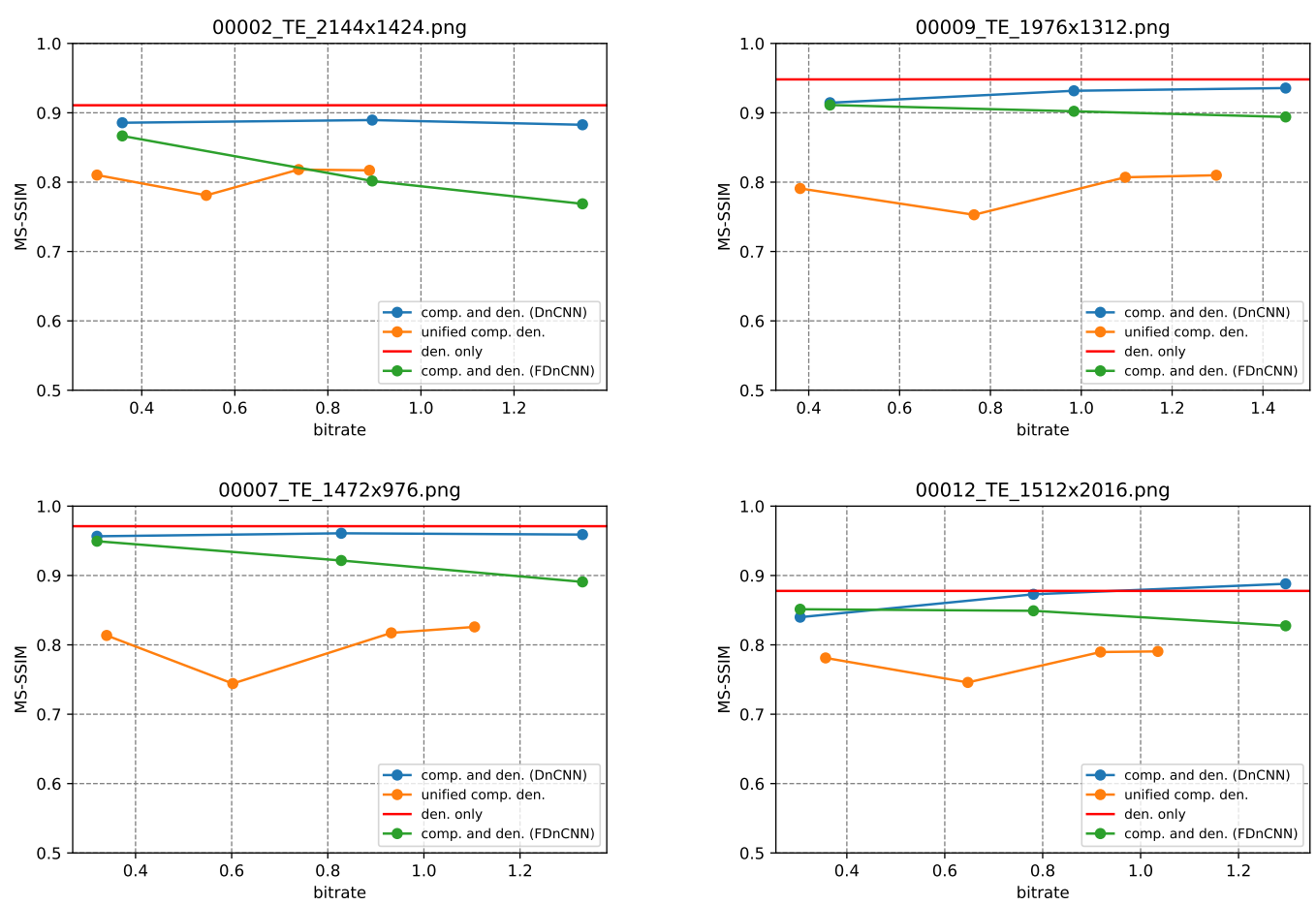

Figure 6: Rate-distortion results for the MS-SSIM objective quality metric on the different compression and denoising pipelines. 

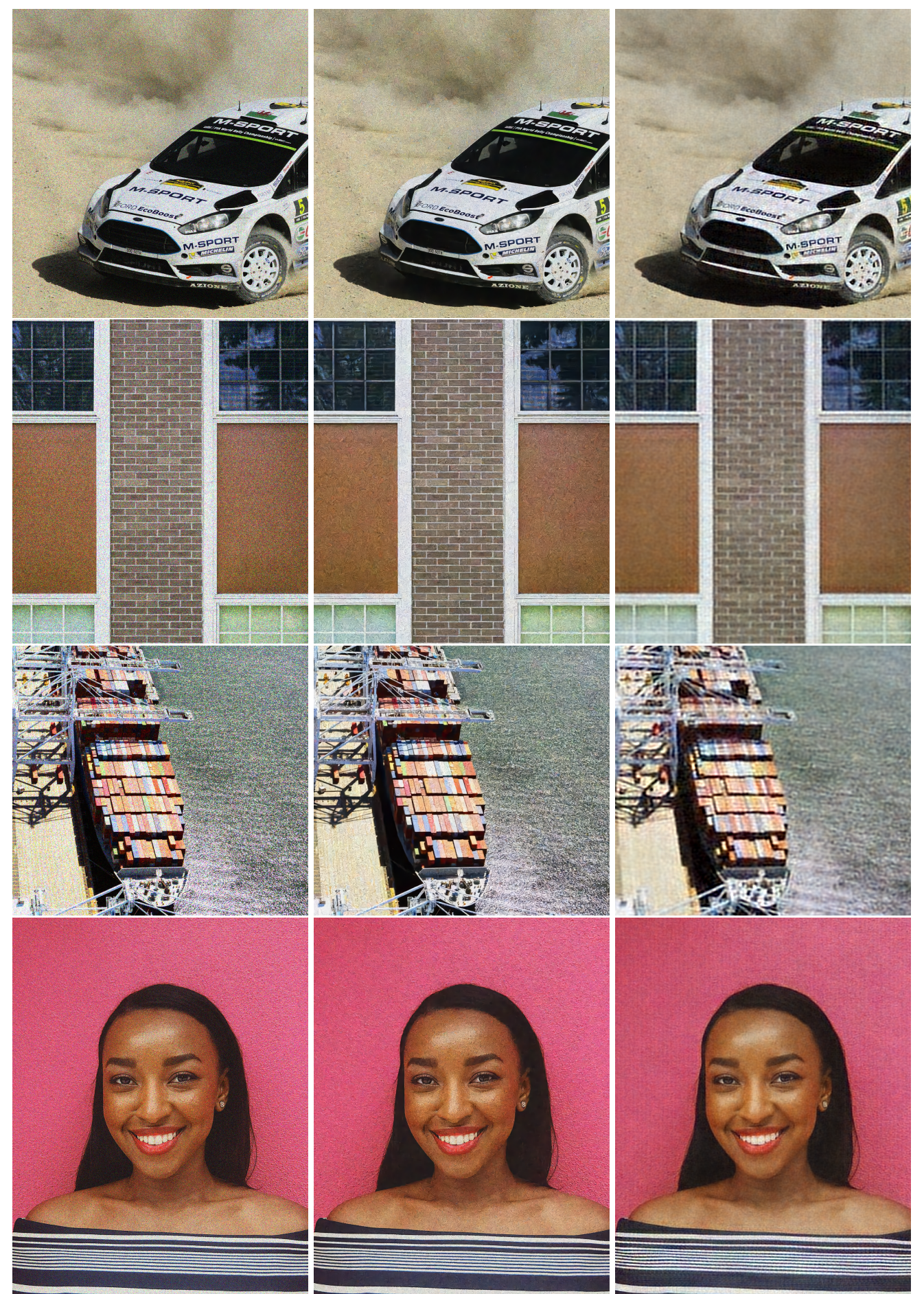

(a) Noisy uncompressed images(b) Compression and denoising

(c) Proposed results

Figure 7: Visual comparison of the results obtained with the different pipelines, at the highest bitrates. Column (a) shows some noisy uncompressed crops of the testset; column (b) shows the crops obtained applying compression and denoising in cascade; column (c) shows the results obtained with the proposed combined compression and denoising architecture. 
The plots show that the best visual results are achieved by the DnCNN denoising network, which indicates an increasing performance with the increase in the bitrate. Moreover, the model is able to achieve the same performance as the ideal case of denoising without compression in several cases.

It can also be observed that, when considering the pipeline of compression and denoising using the FDnCNN in cascade, no gain in performance is achieved at the highest bitrates, and in some cases the performance is lower than the lowest bitrate. This might be caused by the fact that the compression changes the strength and distribution of the noise, and therefore the performance of the denoising network is decreased. Moreover, the compression network tends to introduce blurring artifacts in the images, especially at the lowest bitrates, which can be considered a form of denoising method.

The proposed combined compression and denoising method, instead, is able to achieve similar results to the pipeline with compression and denoising in cascade at the highest bitrates. Some visual results of images at the highest bitrate are shown in Figure 7. The visual results show that both the pipeline with compression and denoising in cascade and the combined method present some visual artifacts in the compressed and denoised images. In particular, in the results obtained with the pipeline of compression and denoising in cascade, the noise is still visible in some parts of the images, while the proposed combined pipeline introduces blurriness and a shift in colors. As an example, in the image shown in the first raw of Figure 7, it is possible to observe that the pipeline of compression and denoising in cascade does not achieve good denoising performance in the background of the image, while the proposed combined method present improved visual results. On the contrary, the compression and denoising in the cascade pipeline gives better visual results in the front part of the car compared to the proposed combined method.

\subsection{Computational complexity analysis}

One of the main advantages of having a combined compression and denoising architecture is in the improvement of computational complexity. The gain in performance is shown in Table 1. In particular, the run time for the tasks of compression and denoising, along with the performance of the combined model are reported.

As introduced above, different implementations of the denoising architecture were tested. In particular, for the DnCNN the Matlab implementation, proposed by the authors, was tested on a MacBook Pro with $2.3 \mathrm{GHz}$ 8-Core Intel Core i9 processor, and without a graphics processing unit (GPU). The FDnCNN has been tested on a machine with a GeForce GTX 1070 graphic card. The results show, as expected, that the average running time on the images of the testset for the FDnCNN is considerably lower than the DnCNN. As for the compression task, the model has an average computational time of 20 seconds in total. For the proposed combined compression and denoising method, instead, the average running time is slightly higher, around 26 seconds in total.

When considering the compression followed by denoising pipeline, and considering the FDnCNN model, running therefore on a GPU, the total running time is about $34 \mathrm{~s}$, which makes the running time of the combined method $23.5 \%$ faster.

Table 1: Results of the computational complexity analysis on the test set for the examined pipelines

\begin{tabular}{|c||c|c|c|c|c|c|}
\hline \multicolumn{7}{|c|}{ Computational complexity } \\
\hline \multicolumn{1}{|c||}{} & \multicolumn{2}{|c|}{ Denoising } & \multicolumn{2}{c|}{ Compression } & \multicolumn{2}{c|}{ Combined method } \\
\hline $\begin{array}{c}\text { Ave. running time } \\
\text { (s) }\end{array}$ & $890 \mathrm{~s}$ & $12.54 \mathrm{~s}$ & $9.9 \mathrm{~s}$ & $11.33 \mathrm{~s}$ & $11.67 \mathrm{~s}$ & $14.17 \mathrm{~s}$ \\
\hline $\begin{array}{c}\text { Total time (s): } \\
\text { best time }\end{array}$ & \multicolumn{3}{|c|}{$34 \mathrm{~s}$} & $26 \mathrm{~s}(-\mathbf{2 3 . 5 \% )}$ \\
\hline $\begin{array}{c}\text { Total time (s): } \\
\text { best visual results }\end{array}$ & \multicolumn{3}{|c|}{$911 \mathrm{~s}$} & $26 \mathrm{~s}(-\mathbf{9 7 \%})$ \\
\hline
\end{tabular}




\section{CONCLUSIONS}

In this paper, a combined architecture for compression and denoising was proposed. The experimental results show that, while achieving similar rate-distortion performance at the highest bitrates, the method is able to reduce computational complexity when compared to a pipeline in which the compression and the denoising tasks are executed in cascade.

By all means, the presented results are preliminary and only demonstrate that such types of approaches are feasible and have an interesting potential. Therefore, further investigations in this topic are needed. As an example, a different architecture including batch normalization layers, or optimizing a different full-reference or no-reference objective quality metric, more suitable for the denoising task, could be explored. In addition, to improve the performance, the pipeline can be specialized for a specific target application, e.g for face detection or face recognition.

\section{ACKNOWLEDGMENTS}

The authors would like to acknowledge support from the Swiss National Foundation for Scientific Research project entitled "Advanced Visual Representation and Coding in Augmented and Virtual Reality" under grant number 200021_178854.

\section{REFERENCES}

[1] Ballé, J., Laparra, V., and Simoncelli, E. P., "End-to-end optimized image compression," in [5th International Conference on Learning Representations, ICLR 2017], (2017).

[2] Ballé, J., Minnen, D., Singh, S., Hwang, S. J., and Johnston, N., "Variational image compression with a scale hyperprior," in [International Conference on Learning Representations], (2018).

[3] Minnen, D., Ballé, J., and Toderici, G. D., "Joint autoregressive and hierarchical priors for learned image compression," Advances in Neural Information Processing Systems 31, 10771-10780 (2018).

[4] Agustsson, E., Tschannen, M., Mentzer, F., Timofte, R., and Gool, L. V., "Generative adversarial networks for extreme learned image compression," in [Proceedings of the IEEE/CVF International Conference on Computer Vision], 221-231 (2019).

[5] Mentzer, F., Toderici, G. D., Tschannen, M., and Agustsson, E., "High-fidelity generative image compression," Advances in Neural Information Processing Systems 33 (2020).

[6] Torfason, R., Mentzer, F., Agustsson, E., Tschannen, M., Timofte, R., and Van Gool, L., "Towards image understanding from deep compression without decoding," in [International Conference on Learning Representations], (2018).

[7] Zhang, Q., Liu, D., and Li, H., "Deep network-based image coding for simultaneous compression and retrieval," in [2017 IEEE International Conference on Image Processing (ICIP)], 405-409, IEEE (2017).

[8] Toderici, G., O'Malley, S. M., Hwang, S. J., Vincent, D., Minnen, D., Baluja, S., Covell, M., and Sukthankar, R., "Variable rate image compression with recurrent neural networks," in [International Conference on Learning Representations, ICLR 2016, San Juan, Puerto Rico], (2016).

[9] Toderici, G., Vincent, D., Johnston, N., Jin Hwang, S., Minnen, D., Shor, J., and Covell, M., "Full resolution image compression with recurrent neural networks," in [Proceedings of the IEEE Conference on Computer Vision and Pattern Recognition], 5306-5314 (2017).

[10] Theis, L., Shi, W., Cunningham, A., and Huszár, F., "Lossy image compression with compressive autoencoders," in [International Conference on Learning Representations, ICLR 2017, Toulon, France], (2017).

[11] Ballé, J., Laparra, V., and Simoncelli, E. P., "End-to-end optimized image compression," in [International Conference on Learning Representations, ICLR 2017, Toulon, France], (2017).

[12] Jain, V. and Seung, S., "Natural image denoising with convolutional networks," Advances in neural information processing systems $\mathbf{2 1}$ (2008).

[13] Zhang, K., Zuo, W., Chen, Y., Meng, D., and Zhang, L., "Beyond a gaussian denoiser: Residual learning of deep cnn for image denoising," IEEE transactions on image processing 26(7), 3142-3155 (2017).

[14] Zhang, K., Zuo, W., and Zhang, L., "Ffdnet: Toward a fast and flexible solution for cnn-based image denoising," IEEE Transactions on Image Processing 27(9), 4608-4622 (2018). 
[15] Tai, Y., Yang, J., Liu, X., and Xu, C., "Memnet: A persistent memory network for image restoration," in [Proceedings of the IEEE international conference on computer vision], 4539-4547 (2017).

[16] Gu, S., Timofte, R., and Van Gool, L., "Multi-bin trainable linear unit for fast image restoration networks," arXiv preprint arXiv:1807.11389 (2018).

[17] Foi, A., Trimeche, M., Katkovnik, V., and Egiazarian, K., "Practical poissonian-gaussian noise modeling and fitting for single-image raw-data," IEEE Transactions on Image Processing 17(10), 1737-1754 (2008). 